\title{
Laurenz Sondereggers Briefe an Arnold Baader, den Redaktor des „Correspondenzblattes für Sdhweizer Ärzte”
}

Von $\mathrm{H}$ e in ri ch B u e ß

«Kaum ein anderer Arzt unserer Zeit hat mit gleicher Wärme der Überzeugung die Sache dieses weltbürgerlichen Humanismus vertreten und den großen Zukunftsberuf unserer Medizin mit gleicher Begeisterung aufgefaßt wie der Schweizer Sonderegger in seinem kürzlich erschienenen bedeutenden Werke.» Diese Worte widmet der dänische Medizinhistoriker J a k o b J u li u s Pe tersen (1840-1912) den "Vorposten der Gesundheitspflege» des St. Galler Arztes Jakob Laurenz Sonderegger. Ja, er hält dieses für die Erziehung des Volkes bestimmte Buch für so bedeutend, daß er damit seine Darstellung der «geschichtlichen Entwicklung der medizinischen Therapie» (Kopenhagen 1877) abschließt. Tatsächlich hat das Werk Sondereggers weit über die Landesgrenzen hinaus Verbreitung gefunden.

Aber auch Sonderegger als Persönlichkeit lebt heute in den Herzen der älteren Ärzte fort. Der diesjährige Schweizerische Ärztetag versammelte sich in St. Gallen, und der Präsident der Verbindung der Schweizer Ärzte gedachte in erhebenden Worten seines großen Vorgängers. Ob die Wahl des Tagungsortes durch die Persönlichkeit Sondereggers bestimmt war, entzieht sich meiner Kenntnis. Jedenfalls aber hat der Schweizerische Ärztestand diesen Sommer besonderen Anlaß, des mannhaften Vorkämpfers für seine Interessen zu gedenken. Hat sich doch am 20. Juni zum 50. Mal der Tod Sondereggers gejährt.

Es war ein merkwürdiges Zusammentreffen, daß ausgerechnet dieses Frühjahr Herr Kollege Carl Haffter, Sekundärarzt in der Friedmatt, Basel, mir die Briefe Sondereggers an Arnold Baader übergab, gerade als die anderswo ${ }^{1}$ erschienene kurze Würdigung abgeschlossen war. Denn diese bisher unveröffentlichten Dokumente lassen nicht nur Sondereggers Eigenart aufs Neue in schönstem Licht hervortreten, sondern sie sind auch für die Geschichte des Medizinalwesens und des Ärztestandes in der Schweiz von hohem Wert.

Eine kurze biographische Einführung soll der Wiedergabe der Briefe vorangestellt werden.

1 Schweiz. med. Wschr. 27, $1141 \mathrm{ff.} \mathrm{(1946);} \mathrm{daselbst} \mathrm{auch} \mathrm{die} \mathrm{Bibliographie} \mathrm{der}$ Schriften von und über Sonderegger. 
Jakob Laurenz Sonderegger wurde am 22. Oktober 1825 im St. Galler Rheintal geboren. Sein Vater war Verwalter des der Familie Custer gehörenden Schlosses Grünenstein bei Balgach. Von früh auf verband den Knaben ein besonders inniges Verhältnis zur tiefgläubigen Mutter. Von ihr mag er die Neigung zu philosophischen Studien - Spinoza und Kant waren seine Lieblinge - und den bewundernswerten Glaubensmut ererbt haben. In Peter Scheitlin (1799-1848), dem tonangebenden Haupt des wissenschaftlichen Lebens in St. Gallen, erhielt er am «Bubenkloster» (dem Obergymnasium der Hauptstadt) einen Lehrer, der ihm die Schätze des Naturreiches zu erschließen wußte. Als Student in Zürich brachte er es mit eiserner Energie zustande, daß er seinen Sprachfehler verlor. So schwach er dem Körper nach damals gewesen sein mag, so vermochte doch Sondereggers von hohen Idealen beseelter Geist seine Kameraden anzuspornen, und es ergab sich von selbst, daß sie ihn zum Wortführer erkoren. $\mathrm{Zu}$ seinen Lehrern trat er auch an den verschiedenen Hochschulen des Auslandes (Würzburg, Wien, Prag, Leipzig) in ein näheres Verhältnis, wie seine von Haffter veröffentlichten Briefe zeigen. Wer hätte auch den impulsiven und frohmütigen Ostschweizer nicht lieb gewinnen sollen!

Nach bestandenem Examen (1850) praktizierte Sonderegger zunächst in Balgach, später in Altstätten und schließlich in St. Gallen, als ihm die beschwerliche Landpraxis zu viel wurde. Ohne Zweifel hätte Sonderegger auch als akademischer Lehrer Bedeutendes geleistet, ein Ziel, auf das er nur schweren Herzens verzichtete. Aber rückblickend dürfen wir wohl sagen, daß Sonderegger der Mann war, wie ihn der schweizerische Ärztestand im jungen Bundesstaat nötig hatte. Innert kurzer Zeit griff seine Wirksamkeit auf kantonalen und dann auf eidgenössischen Boden über. Die immer wieder auftretenden Epidemien von Pocken und Typhus mögen ihn zum erstenmal in diese Richtung gewiesen haben. Mit Wort und Tat nahm er sich der in ungesunden Hütten lebenden Mitbürger an, bald wußte er auch die Blicke der Behörden auf die teilweise erschütternden Verhältnisse zu lenken. Seine hinreißenden Sätze rüttelten die verantwortlichen Männer aus ihrer Ruhe auf. Einer seiner ersten Erfolge war der Bau des Kantonsspitals in St. Gallen. Bald wurde Sonderegger zum Präsidenten des «Ärztlichen Zentralvereins» gewählt (1870). Durch sein einzigartiges Werk, die «Vorposten» (Berlin 1873), lenkte er die Aufmerksamkeit des Bundesrates auf sich, und von da an hatte er die Schweiz auf mehr als einem internationalen Kon- 
greß zu vertreten. Seine «Lebenserinnerungen» wissen davon manches Köstliche zu berichten. In Rom regte er die Gründung eines internationales Sanitätsbureaus an. Der Glarner Fridolin Schuler (1832-1903) sekundierte seinen Freund in glänzender Weise. Ihren gemeinsamen Bemühungen ist die Schaffung des eidgenössischen Gesundheitsamtes (1893) zu verdanken, das den von Sonderegger etwa zwei Jahrzehnte lang geleiteten «Zentralverein» in seinen mannigfachen Aufgaben ablöste. Auch dem Hygiene-Unterricht schenkte er seine ganze Tatkraft und als Examinator sein reiches Wissen. Durch seine vielen kleineren Schriften vermochte Sonderegger manchen Übelstand zu beseitigen. Eine der letzten Bestrebungen galt der Fürsorge für die Tuberkulosekranken.

So gibt es in der ganzen Gesundheitspflege kaum ein Gebiet, das Sonderegger nicht zu bleibendem Dank verpflichtet wäre. Immer fühlte er sich auch zur Jugend hingezogen, und seine Gedanken über das medizinische Studium gehören zum Schönsten, was ein angehender Arzt lesen kann. In makelloser Reinheit suchte er das ärztliche Ethos zu erhalten und über den Kleinkram des Alltags emporzuheben.

Davon zeugen nicht nur die sprühenden Reden an den Ärztetagungen sondern auch die nachstehenden Briefe. Diese sind an seinen Kollegen Arnold Baader (1842-1888; näheres siehe die erste Anmerkung), den Redaktor des «Correspondenzblattes für Schweizer Ärzte» gerichtet. Diese im Jahre 1870 gegründete Zeitschrift diente der Weiterbildung der praktischen Ärzte und auch der Diskussion über aktuelle Fragen des Ärztestandes. Sie hatte also zwei verschiedene Zwecke zu erfüllen. Diese Tatsache spiegelt sich auch in den hier veröffentlichten Dokumenten. Zum besseren Verständnis sind in einigen Anmerkungen die nötigen biographischen Daten beigefügt. Leider war es trotz zahlreichen Anfragen in Kantons- und Gemeindekanzleien nicht möglich, über jeden der von Sonderegger angeführten Ärzte biographische Daten zu geben.

Auch eine zweite Lücke mußte offenbleiben. Alle Bemühungen, die Briefe der Redaktoren des «Correspondenzblattes» (namentlich von Arnold Baader), auf die sich die vorliegenden Dokumente beziehen, zu erhalten, blieben erfolglos. Weder Dr. Paul Jung in St. Gallen, noch P. D. Dr. G. A. Wehrli, die Teile des Nachlasses Sondereggers besitzen, konnten hier weiterhelfen. Auch die Redaktion der Schweiz. med. Wschr. weiß nichts über den Verbleib dieser Korrespondenz. (Den Herren Drs. Jung, Wehrli und Merkelbach danke ich für ihre Bemühungen.) 
Vielleicht darf im Hinblick auf die nachstehenden Briefe $^{2}$ auch von den Lesern unserer Gedenkworte gesagt werden, was Elias Haffter in seinem Vorwort zur Herausgabe von Sondereggers Selbstbiographie schreibt: "Der Name Sonderegger bleibt in die Herzen seiner zeitgenössischen Ärzte tief eingegraben; mit dem Schwungrad seines hervorragenden Geistes und der Kraft seines gesprochenen und geschriebenen Wortes hat er wohl jedem, der mit ihm in Berührung getreten, manchen frischen und idealen Impuls erteilt... Denn wer immer das herrlich geschriebene autobiographische Lebensbild gelesen, für den ist der Verfasser kein Fremdling mehr, sondern ein dem Herzen nahestehender Mensch, und er wird mit liebevollem Interesse sich in den Briefen weiter und intimer mit ihm beschäftigen.»

Herrn Dr. A. B a a d e ${ }^{1}$

\section{St. Gallen d 12. März 1877}

Verehrter Herr College!

Vor Allem: gute Genesung! und einen herzlichen Grüß Gott!

Ich hätte Ihnen ohne Burkhardts² Auftrag diese Arbeit nicht geschickt, sondern sie an Burkhardt zurückgegeben.

Mich hat die Schrift sehr betrübt. Vogt ${ }^{3}$ erklärt, noch keine Meinung zu

2 Die Wiedergabe der Briefe erfolgt ohne jede Änderung oder Weglassung. Lediglich die Abkürzungen für «und» («\&», «u.» usw.) wurden ausgeschrieben, weitere Korrekturen unterblieben jedoch. Die Briefe sind in meist gut leserlicher und zierlicher deutscher Fraktur niedergeschrieben.

${ }_{1}$ Der Empfänger sämtlicher hier vorliegender Briefe ist $\mathrm{A} \mathrm{rnold} \mathrm{Ba} \mathrm{a} \mathrm{der,}$ (geb. 16. Febr. 1842 in Gelterkinden, gest. 25. Febr. 1888 in Basel), einer der bedeutendsten Baselbieter Ärzte. Baader war von 1872 an Redaktor des «Correspondenzblattes», zuerst als Arzt in Gelterkinden, wo schon mehrere Vorfahren im gleichen Beruf tätig gewesen waren, von 1879 an in Basel. Er schrieb besonders über militärmedizinische und geburtshilfliche Themata. Vgl. auch Anm. 43.

2 A 1 bert Burckhardt-Merian (1843-1886), der erste Redaktor des «Correspondenzblattes», lehrte zunächst Kinderheilkunde und Chirurgie, von 1879 an Ohrenheilkunde, wohl als erster Extraordinarius für dieses Fach in der Schweiz. Vgl. auch Anm. 29.

3 A d olf Vogt (1823--1907), der Bruder Carl Vogts und des Juristen Gustav Vogt, ist eine der unrühmlichen Gestalten unter den akademischen Lehrern an schweizerischen Hochschulen. Ohne irgendwie dafür befähigt zu sein, versah er von 1876-1892 die Professur für Hygiene an der Universität Bern. (Vgl. über ihn Rich. Feller, Die Universität Bern, S. 308-310, 4.11 f.) Er ist namentlich bekannt als Gegner der Pockenimpfung (in diesem Sinn ist die Angabe im «Histor.-biogr. Lexi- 
haben, behandelt aber die besten Leute, Virchow Hebra \& Comp. als Thoren und Betrüger, tischt uns förmliche Sophismen und Trölereien auf und rükt mit der ganzen Geschichte heraus, während Brukner ${ }^{4}$ Schuster und Zopfi im Kampfe liegen - und verstärkt nur diese! Es gèniert den Arzt nicht, ein Experiment mit dem Volke zu machen und gèniert den akademischen Lehrer nicht, mit einer Herostrates-Arbeit sein Amt anzufangen; er merkt nicht einmal, daß er ganz und gar auf die unfruchtbare Haide läuft, auf welcher Hamernjk und Engel ${ }^{5}$ und so manche große «Mathematiker» umgekommen sind.

Wenn es sich um die Verbesserung der Impfung handelt ist die schneidenste Kritik am Platze, - im jetzigen Moment begeht Vogt in aller Form eine Frivolität, ja eine ärztliche Sünde, wenn er losschlägt. Weiß er denn wirklich so genau, daß er recht hat, daß er es darauf hin wagen kann, die mühsam errungene Impfung zu zertrümmern?

Können Sie ihn zur Verschiebung bewegen ist's gut. Will er nicht verschieben, so wissen wir, daß er nicht die Wissenschaft, sondern die Compagnie Brukner fördern will.

Ich weiß, daß ich zu einem Freunde Vogt's spreche und berechtige Sie, ihm wenn Sie wollen, diese Zeilen zu geben. Ich achte Vogt und halte ihn für unendlich nobler als er oft scheint - aber dieser kühle Mephistopheles mit dieser legèren Manier macht mich schaudern. Wir müssen aufbauen, nicht niederreißen!

In steter Hochachtung und Ergebenheit Ihr

Dr. Sonderegger

An die Redactoren des Schweiz. Corr. Blattes für Ärzte die Herren Doct. Burkhardt Merian und Dr. Baader

St. Gallen d 13. März 1877

Verehrte Herren!

Sie haben mir das Vertrauen bewiesen, eine Einsendung unsers Herrn Collegen Prof. Dr. Vogt Impfstatistik betreffend, zur Einsicht vorzulegen und ich

kon der Schweiz» richtigzustellen), wie auch der vorliegende Brief deutlich zeigt. Um welche «Schrift» Vogts es sich hier handelt, deutet der zweite Brief an. Wahrscheinlich waren es Teile der später (Bern 1881) im Druck erschienenen Schrift: «Der alte und der neue Impfglaube».

${ }^{4}$ Gemeint ist wohl Theophil B r u ckner (1821-1896), der als Homöopath großes Ansehen genoß. Ein Schweizer Arzt namens Schuster, der hier in Frage käme, ist mir nicht bekannt. Um den deutschen Mediziner dieses Namens (Ludwig Schuster) wird es sich hier wohl nicht handeln. Auch über «Zopfi» ließ sich nichts Einschlägiges finden.

5 J o s e p h H a mernik (1810-1887), Prof. der medizinischen Klinik in Prag, wo Sonderegger seine Vorlesungen hörte. «Engel», wahrscheinlich der pathologische Anatom J osef Engel (1816-1899), der auch zu den Lehrern Sondereggers gehörte und mit dem er freundschaftlich korrespondierte (s. Sonderegger, Selbstbiographie und Briefe, S. 303-309). 
halte mich verpflichtet Ihnen folgende Betrachtung zu freundlicher Überlegung zu empfehlen.

Der eigentliche Präsident und Schwerpunkt der schweizer. Ärzte-Vereinigung ist das Correspondenzblatt. Dieses Band hält weit fester als die Versammlungen. Es muß, in seinem Interesse wie zum Wohle der Schweiz. Ärztevereinigung, der Ausdruck der jeweilen herrschenden Ansichten sein.

Nach dem Ergebnisse der Impfabstimmung 6 ist die Stellung der Schweiz. Ärzte in dieser Frage eine sehr scharf ausgesprochene und es ist weder gerecht noch klug, jetzt, während Freunde und Gegener der Impfung vor den Eidg. Räten erscheinen, Ansichten zu verfechten, welche $96 \%$ der Ärzte gegen sich haben.

Ist die Impfung, vorläufig wenigstens gesichert, so wird eine Kritik im Style der Vogt'schen nur wohltätig wirken, gegenwärtig aber dient sie nicht der Wissenschaft, sondern wider Willen, einer gemeinen Agitation, weßhalb ich vorschlage, die Vogtsche Arbeit einstweilen zurückzulegen. Wir haben auch dem Ausland gegenüber unsere Ehre zu wahren und ein Keller ${ }^{7}$ wiegt mir, von aller persönlichen Ansicht abgesehen, die deutschen französischen und englischen "Impfgläubigen» nicht auf. Mißverstehen Sie mich nicht, werthe Freunde, aber wir müssen unsere großartige Mehrheit beisammenzuhalten den Muth haben!

In steter Hochachtung und Ergebenheit

Ihr Dr. Sonderegger

Ps. der Ärzte Commiss.

Titl Redaction des Corr Blattes.

Herrn Drr. Burkhardt-Baader

St. Gallen d 22 Mrz 1877

Verehrte Herren Collegen!

Sie erweisen mir zu viel Aufmerksamkeit und ich bin ganz zufrieden, wenn Sie meine Briefe, die Producte einer ehrlichen Aufregung, nicht übel aufnehmen. Sie sind von mir unabhängig und wissen besser was dem werthvollen Organ der Schweiz. Ärzte frommt als ich.

Mein letztes Wort in dieser Sache ist folgendes:

Ich würde Vogt anfragen, ob er mit seiner Arbeit Verbesserung der Impf-

${ }^{6}$ Bei dieser «Abstimmung» handelt es sich um eine Rundfrage innerhalb der schweizerischen Ärzteschaft, da die erste Volksabstimmung über das «Epidemiengesetz», in der es zur wuchtigen Verwerfung der hauptsächlich von Sonderegger befürworteten Vorlage kam, erst im Jahre 1882 durchgeführt wurde. Vgl. Sondereggers «Petition der Impffreunde». St. Gallen 1877.

7 Welcher der zahlreichen Ärzte dieses Namens sich näher mit der Impffrage befaßt hat, geht aus den mir zur Verfügung stehenden Werken nicht sicher hervor. Vielleicht handelt es sich um den Landsmann Sondereggers Alo is Keller (1829-1895) aus dem Bezirk Uznach. 
statistik oder Unterstützung der Impfgegner anstrebt? In ersterem Falle wäre es angezeigt, die Arbeit zurückzulegen bis die Frage vor den Bundesbehörden entschieden ist; im letzteren Falle aber werden die Redactoren ihrem Collegen das Wort ohne weiteres geben und es dem Professor der Hygiene überlassen, wie er sich damit zur öffentlichen Meinung der schweizerischen Ärzte stelle.

Der gute Mann, den ich für besser halte als er auszusehen beliebt, muß Farbe bekennen und wir müssen genau wissen wie wir uns in dieser Frage zu ihm stellen.

Seien Sie mir in steter Hochachtung und Freundschaft gegrüßt!

Ihr

$$
\text { Dr. Sonderegger }
$$

Mein lieber Herr College!

St. Gallen d $29 \mathrm{Mrz} 1877$

Mitfolgende Zeilen bringen Ihnen keine Geschäfte; ich komme nur, Ihnen die Hand zu drücken und Ihnen grüß Gott zu sagen und mich mit Ihnen zu freuen, daß es Ihnen wieder besser geht! Ich habe so oft Ihrer gedacht, denn ich habe, bei schlechterer Constitution, und ebenfalls bei äußerst strapaziöser Landpraxis, ähnliche Erlebnisse durchgemacht und bin auch für Wochen lang ins Bette oder an Curorte gegangen zu Zeiten, da ich mir nicht erlaubte, für mein Vergnügen eine Stunde auszuruhen. Lassen Sie die unvollkommene Erde hinter sich, wenn Sie auf die glückliche Insel Montreux oder Gersau reisen; lassen Sie sichs nicht zu Herzen gehen, daß Sie Vieles versäumt und verlassen haben und genießen Sie mit so viel Ruhe und Muße die unfreiwilligen Ferien, als es Ihr lebendiger Geist nur erlaubt!

Nebenbei bitte ich um Verzeihung, daß ich in Ihre Redactionsgeschäfte hineinzupfuschen versuche. Ich fühle Reue und verspreche Besserung!

Vogt ist in den Impfgegner Verein als Mitglied eingetreten; vermutlich um sich die gerühmte "wissenschaftliche Objektivität» zu wahren. Es thut mir um den Mann so leid wie um die Sache. Nun Gottbefohlen! Ich wünsche Sonnenschein in jeder Beziehung und grüße in steter Freundschaft und Hochachtung,

Ihr

Dr. Sonderegger

Werther Herr

St. Gallen d 17 März 1879. und Freund!

Meine warme, herzliche Condolenz beim Hinschiede Ihres hochachtbaren Vaters. ${ }^{8}$ Ich werde den merkwürdigen Mann niemals vergessen, der mit der

${ }^{8}$ Der Brief ist an Arnold Baader adressiert, dessen Vater $\mathrm{J} \mathrm{o} \mathrm{h} \mathrm{a} \mathrm{n} \mathrm{n} \mathrm{J} \mathrm{a} \mathrm{k} \mathrm{o} \mathrm{b}$ B a a d e r (geb. 4. April 1810) am 16. März 1879 starb. (Dem Staatsarchiv Liestal spreche ich für diese Angaben meinen besten Dank aus.) J. J. Baader darf als 
Energie eines Feldherren die Liebenswürdigkeit eines Kindes vereinte! Das war ein Mann! Gott gebe Ihnen Trost und Gesundheit, und Befriedigung in Ihrem neuen Wirkungskreise!

In steter freundschaftlicher Hochachtung

Ihr Dr. Sonderegger

Herrn Drr. Burkhardt Merian und Baader

Liebe Freunde! ${ }^{9}$

St. Gallen d 15 Nov 83.

Es ist ein schlechtes Unterfangen Freunden zu opponieren und einem zugleich klugen und begeisterten Manne die kalte Teufelsfaust der Prosa und der Philisterei auf den Hals zu setzen. Dennoch sei es; der Zweck heiligt auch diese Mittel! Ich mache in aller Ruhe folgende Einwürfe.

1. Von Berathungen und Berichten bis Febr. 84 kann keine Rede sein; bis dann versammelt sich keiner aller cantonalen Ärztevereine.

2. Wenn sie sich versammeln und discutieren, gehts erst wieder $1-2$ Sitzungen, d.h. ein Jahr. Octroiren können wir die Statuten nicht und die parlamentarische Behandlung geht lange, multa capita et multi sensus! Unterdessen müssen wir ein ordentliches Provisorium haben!

Ich will überhaupt lieber mit einem kleinen Programme anfangen als mit einem ausgearbeiteten, wenn auch sehr guten, System.

3. Das Obligatorium wird schwieriger je länger man es anschaut. Die ärztl. Cantonalgesellschaften sind sehr lose Verbände ohne viel Autorität über den Einzelnen oder seinen Geldbeutel; wer nicht will den kann Niemand zwingen und aus dem cantonalen Verein gestoßen wird er deswegen auch nicht.

4. Viele Cantonalvereine werden verbleiben und das Ganze wird ein Stückwerk, eine sehr knarrende Maschine mit viel Rädern und wenig Nutzaffekt.

5. Nehmen wir einzelne Mitglieder zu dem freiwilligen Verband mit fixen Beiträgen? Dann bekommen wir einen Verein im Verein. Die Namen da und dort deken sich oft, oft aber auch nicht.

6. Jedem Obligatorium, und wäre es auch freiwillig angetreten, steht ein Recht gegenüber und wir mögen uns winden und drehen wie wir wol-

der bedeutendste Baselbieter Arzt gelten, er ist um die Entwicklung des ärztlichen Standes in der Schweiz hochverdient, regte als Präsident der medizinischen Gesellschaft von Baselland die Gründung des «Ärztlichen Zentralvereins» an (1865) und ist auch an der Gründung des von Edwin Klebs geschaffenen «Correspondenzblattes» namhaft beteiligt. Als kantonaler Politiker und als Nationalrat setzte er sich kräftig für hygienische und soziale Bestrebungen ein.

9 Den größten Anteil an der Gründung der «Hülfscasse für Schweizer Ärzte» haben Alb. Burckhardt-Merian, der am 29. Oktober 1881 eines der beiden Projekte einreichte, und Arnold Baader, dessen Vortrag «Die Hülfscasse für Schweizer Ärzte» (in Basel 1883 im Druck erschienen) die praktisch gangbarsten Vorschläge enthielt. Als Gutachter wirkte auch der Basler Physiker und Philanthrop Fritz Burckhardt-Brenner (s. nächste Anm.). 
len, so gründen wir damit eine Art Rentenanstalt etc. Ein Obligatorium ohne positive Ansprüche ist gar nicht denkbar, ausgenommen bei einem «freiwilligen Armenverein». Das Obligatorium macht aus der Freundesspende eine Armensteuer in gesetzlichster Form.

7. Wir müssen also mit dem jetzigen System eine Zeitlang fortfahren, bis sich Bedürfnisse zu Weiterem zeigen. Kommen sie schon aus den cantonalen Berathungen des Baaderschen Entwurfes, - desto besser; aber ich bin vorläufig Pessimist.

Dann möchte ich kurz sein, nicht die schöne Baadersche Arbeit im Circular theilweise wiederholen; ich möchte keine cantonale Geberstatistik. Dieser Peitschenhieb kommt später, wenn der Pegasus faul wird. Wir haben ja jetzt doch so viel, wie wenn jeder schweizer Arzt seine obligatorischen Fr. 5. - gezahlt hätte. Erlahmen, desertiren etc können diese obligator. Fr.-5-Männer ganz so gut wie die freiwilligen Fr.-20-50-Männer.

Kurz, bis die Sache die cantonalen Vereine passirt hat, würde ich beim jetzigen Modus verbleiben und unser Program in der vorgeschlagenen Weise geben; mutatis mutandis, ich halte nicht auf meinem Wortlaute und bin zu realen und formalen Änderungen stets sehr gerne bereit.

Nun bin ich zu Ende, ärgert Euch nicht, verzeiht mir und berichtet über Eure Meinung.

Mit herzlichen Grüßen Euer

Dr. Sonderegger

An die Herren Professor Fritz Burckhardt-Brenner ${ }^{10}$ und Physicus Lotz; ${ }^{11}$ in Basel

Verehrte Herren!

St Gallen d 19 März 1884

Im Vertrauen auf Ihre so vielfach erprobte Gemeinnützigkeit wagen wir es, Sie hiermit zu bitten, daß Sie der Hülfs-Casse für Schweizerische Ärzte Ihre Einsicht und ihre Autorität leihen und das Amt von Rechnungsrevisoren übernehmen möchten.

Die noch sehr kleine und von Hrn. Dr. A. Baader sorgfältig verwaltete Casse wird Ihnen wenig Arbeit verursachen, aber diese Arbeit wird zur Beruhigung

10 Professor Fritz B ur ckhardt-Brenner (1830-1913), Physiker, Mathematiker, Schulmann und Politiker, erwarb sich durch sein Eintreten für das Medizinalkonkordat, durch die Gründung der «Allgemeinen Krankenpflege Basel» und durch seine Betätigung in der Verwundetenfürsorge auch bei den Ärzten großes Ansehen. Dr.med.h.c. Basel 1908. Siehe die schöne Würdigung von Gottl. Imhof im Basler Jahrbuch 1914, (S. 1-42)!

11 The ophil Lotz (1842-1905), Kantonsphysikus 1884-1908, einer der eifrigsten Befürworter der Pockenimpfung und erfolgreicher Pionier auf hygienischem Gebiet, namentlich in der damals noch in den Anfängen steckenden Seuchenbekämpfung. 
des Cassiers und zum Credit des Unternehmers wesentlich beitragen. Die Garantie für die Casse haben die Mitglieder der Schweiz. Ärzte Comm., außer den Unterzeichneten die Herrn Prof Kocher (Bern) ${ }^{12}$ Prof. D'Espine (Genf) ${ }^{13}$ Dr. Castella (Freiburg) ${ }^{14}$ Dr. Sigg (Andelfingen) ${ }^{15}$ und Hürlimann Dr. (Unter Ägeri) ${ }^{16}$ solidarisch übernommen.

Indem wir auf Ihre gütige Gewährung unserer Bitte hoffen, danken wir Ihnen zum voraus und versichern wir Sie unserer ausgezeichneten Hochachtung und Ergebenheit.

\author{
Im Namen der schweizerischen Ärzte Commission \\ Der Praeses: \\ Dr. Sonderegger \\ der Schriftführer: \\ Prof. Burckhardt-Merian
}

Wenn ich der Hilfskasse für schweizerische Ärzte einen kleinen Dienst erweisen kann, so bin ich hiezu bereit.

$$
\text { Fr. Burckhardt }
$$

Th. Lotz.

1884. III. 22.

\title{
Gleichfalls
}

22. III. 84

Würde vorschlagen Fritz Bdt + Lotz einzuladen ein Tag zu bestimmen wo ich (?) ihnen bei Fritz Bdt die Rechnung vorlegen könnte.

A. B.

\section{Lieber Freund!}

d. 3. Juni 86

Ihr Brief macht mich ganz unglücklich. Wenn ich der Teufel wäre, würde

12 The od or Kocher (1841-1917), der schon damals weitbekannte Chirurg in Bern.

${ }^{13}$ Ad olphe d'Espine (1846-1930), Prof. der "pathologie interne» in Genf seit 1876 und Mitverfasser des grundlegenden «Manuel des maladies de l'enfance» (1877), Mitglied des Komitees des Internationalen Roten Kreuzes. Nekrolog s. Rev. méd. Suisse rom. 50, 1930, S. 681-686.

${ }^{14}$ Felix Castella (1836-1901), mehr als dreißig Jahre lang Arzt am Großen Bürgerspital in Freiburg i. U., namhafter Militärarzt.

15 Nach der Mitteilung der Gemeindekanzlei in Andelfingen handelt es sich um den Bezirksarzt Joh. Hch. Sigg (geb. 23. Mai 1830, gest. 4. Febr. 1912).

16 J o s e ph H ürlima n n (1851-1911), dem verbreiteten Walchwiler Geschlecht angehörender Arzt, der das Kindersanatorium in Unterägeri gründete (1881), später durch historische Forschungen hervortrat und als Hygieniker auch im politischen Leben seines Kantons eine Rolle spielte. 
ich meinem Todtfeinde genau so verordnen. Es ist entsetzlich, wie frivol Örtel ${ }^{17}$ generalisiert und als Chablone gebraucht wird. Horner ${ }^{18}$ hat letzten Sommer auch sein Theil Örtel-Dilettantismus über sich ergehen lassen müssen.

Protokoll liegt hier; Brief an die Revisoren abenfalls. Das Epid. Gesetz sollte auf 15. Juni a ls Spitze Artikel im Corr Blatte erscheinen. Res capitalis! Selbstverständlich ändern Sie am Protokolle, was Sie wollen, ausgenommen den Passus über Epid. Gesetz. Diesen habe ich mit Deucher ${ }^{19}$ vereinbart. Die Situation ist etwas hart.

Gsellfels $^{20}$ anlangend haben S i e ganz Recht, aber: Sie haben just die blödeste Stelle herausgelangt und ferner: wo haben wir Besseres? Etwa Meyer Ahrens ${ }^{21}$ mit seinen Gedichten? Gsellfels dichtet zwar auch, aber doch "wissenschaftlicher».

Vielleicht finden Sie mich in den Basl Nachrichten pro Pasteur contra Rochefort, ${ }^{22}$ d. h. contra den «infamigten» Advocaten der consequent das Blatt mit Sottisen gegen die Medicin vergiftet.

Grüßen Sie mir Burkhardt sehr und bewahren Sie ihn vor Tollkühnheiten!

Von Herzen Ihr

Dr. Sonderegger

N. B. Deucher ist mit dem Abdrucke meines Entwurfes ebenfalls einverstanden.

17 Gemeint ist wohl Max J oseph Oertel (1835-1897), der bekannte Begründer der nach ihm benannten Kurmethode ( $($ O. sche Terrainkur»), die in seinem Werke «Therapie der Kreislaufstörungen» (1884) theoretisch dargelegt ist und in der Aera vor der Standardisierung der Digitalis große Erfolge zeitigte.

18 Friedrich Horner (1831-1886), der bekannte Augenkliniker in Zürich. Er litt schon längere Zeit an schwerer Arteriosklerose, die auch zu seinem frühen Tode an Apoplexie und Niereninsuffizienz führte.

19 A d o lf De u c her (1831-1912), war als ehemaliger Arzt in Steckborn und Frauenfeld am 10. April 1883 in den Bundesrat gewählt worden, dem er bis zu seinem Tode angehörte. Deucher erwarb sich um den Ausbau der Sozialversicherung, den Arbeiterschutz und um die Volksgesundheit große Verdienste.

${ }_{20}$ 《 G sellfels», eigentlich Johann Theodor Gsell, bekannt unter dem Namen Gsell Fels (1818-1898), vielseitiger Schriftsteller auf medizinischem Gebiet, verfaßte zahlreiche Veröffentlichungen über balneologische Themata.

21 Konrad Meyer-Ahrens, (1813-1872) ist der bedeutendste Medizinhistoriker Zürichs im 19. Jahrhundert und widmete sich als Arzt und Forscher besonders der Balneologie («Die Heilquellen und Kurorte der Schweiz», 1860).

22 L o u is Pas te ur (1822-1895), der berühmte Chemiker und Schöpfer der Bakteriologie; Vi c t or Henri, Gra fvon R o chefort - L u ça y (1830-1913), französischer Journalist, ein Führer der republikanischen Richtung im 2. Kaiserreich. 
Lieber Freund!

Ist Burkhardt in Ragaz? Feurer ${ }^{23}$ schreibt Ihnen nun sein Manuskript. Ich bitte Sie inständig, schiken Sie es ihm n i c h t, sondern nehmen Sie die Arbeit bald auf; es war, nach Cramer, ${ }^{24}$ die beste im ganzen Tage, sehr viel wohlwollender als vieles von mir, was Sie so gütig aufgenommen. Ich möchte Feurer dem Corr. Blatte gewinnen, er ist sensibel, aber ein solider und gescheidter Mensch und ehrlich und wohlwollend wie Wenige; er verdiente Baader zu heiBen, Vater oder Sohn, gleichviel, den «Geist» hat er: «Händ Sorg zue'nem, s'isch s'Chrüz wirth's Sohn von Ägerkingen»!

$\mathrm{Ihr}$

Sonderegger

\author{
Institut Wiget \\ Rorschach \\ St-Gall — Suisse
}

Lieber Freund!

D 10 Septbr 86

Darf ich Sie bitten, im Corr.-Blatte die Versammlung des ärztl. Central Verein nach Olten a f den 30 . October (Samstags) einzuberufen. Ein bestimmtes Programm kann ich noch nicht geben. Künden Sie einstweilen den Tag in leicht sichtbarer Weise an.

Dann - erschrecken Sie nicht! - habe ich ein Manuskript von 24 Folioseiten, die Übersetzung eines preisgekrönten Essay «über Desinfektion» von Sternberg, ${ }^{25}$ der ersten amerikanischen Autorität in diesen Dingen und eines wissenschaftlichen wie auch persönlichen Freundes von Robert Koch. ${ }^{26}$ Vielleicht finden Sie, die Arbeit passe besser in die «Blätter für Gesundheitspflege». Leider herrscht aber auch in ärztlichen Kreisen noch so viel Unklar-

${ }^{23}$ G o t t li e b F e u rer (1853-1925), Chirurg am Kantonsspital St. Gallen, der später auch im Schweizerischen Ärzteverein und im politischen Leben hervortrat. Vgl. über ihn die in einem besonderen Heft vereinigten Nekrologe (St. Gallen 1925).

${ }^{24}$ Nach dem «Correspondenzblatt» (Bd. 16, 1896, S. 369-379, 414-419) hielt an der Tagung des ärztlichen Zentralvereins» der Zürcher «Prof. Dr. C. Cramer» einen Vortrag «Über Bacterien», ein für jene Zeit äußerst aktuelles Thema. Es muß sich um den Schüler Carl v. Naegelis, den Botaniker Carl Ed. Cramer (1831 bis 1901) handeln, der zahlreiche seiner Arbeiten der Bakteriologie gewidmet hat (im Zusammenhang mit der Typhusepidemie in Zürich).

${ }_{25}$ Der amerikanische Militärarzt G e or g e Mill e r S t e r n be r g (1838-1915) entdeckte gleichzeitig mit französischen und deutschen Forschern den Diplococcus pneumoniae (1882) und verfaßte mehrere Abhandlungen über Bakteriologie und Desinfektion.

${ }^{26} \mathrm{Robert}$ Ko ch (1843-1910), der berühmte deutsche Bakteriologe, mit dem Sonderegger, wie er in seinem «Lebensbild» (S. 75 f.) erzählt, persönlich bekannt war. 
heit in dieser Frage, daß ich den Wurf wagen würde. Urtheilen Sie denn selber und refusieren Sie fröhlich, wenn es nicht gut paßt. Auch das hat keine Eile. Wenigstens ein Trost.

Ich war in Ragaz, wo ich meine invalide Frau untergebracht habe, und gehe jetzt nach München, ${ }^{27}$ wo ich das hygienische Institut ansehen und Ferien machen möchte. Logis: Hôtel Bellevue Carlsplatz.

$\mathrm{Da} 3$ die Deutschen meine, vielerorts sehr energisch abgewiesene Pauke freundlich aufgenommen, ist mir um der Sache willen angenehm. Ich selber soll und will immer mehr in den Hintergrund treten, es müssen neue Leute in die Arena .. Aber so lange unser lieber Burckhardt ${ }^{28}$ krank ist, rüttle ich an gar nichts. Grüße mir ihn warm und herzlich. Es ist ein schweres Unglück, wie für seine Freunde und für seine Disciplin, so auch für den Centralverein, wenn er uns verloren geht. Sollten, wie ich immer noch hoffe, bessere Zeiten kommen, o so schicken Sie mir eine Karte nach München!

Das wäre eine Ferienfreude!

Mit herzlichen Grüßen

Ihr Sonderegger

Lieber Freund!

d 11 Decbr 86

Ich bin rücksichtslos und zudringlich wie ein Sektenprediger, erwarte deß. halb aber auch die Nachsicht, welche man den Schwachen schuldig ist. Die Mittheilung wegen des negativen Schlusses von Willes ${ }^{29}$ Grabrede hat mich

${ }_{27}$ Diese Stelle darf wohl als präzisierende Ergänzung zur Notiz Sondereggers auf S. 76 seiner Aufzeichnungen angesprochen werden, wo er von seinem Münchner Aufenthalt und dem tiefen Eindruck berichtet, den ihm Pettenkofer machte.

28 Von hier an als «Burckhardt» (c-k), sonst immer «Burkhardt» geschrieben.

29 Ludwig Wille (1830-1912), Professor der Psychiatrie in Basel von 1875-1904. Durch die vorliegende Kritik Sondereggers an Willes moralisierender Grabrede wird der über der Diagnose von Burckhardts Krankheit liegende Schleier gelüftet. In den im «Correspondenzblatt» (16, 1886, S. 677-689) abgedruckten Gedenkworten Baaders und der übrigen Freunde schreibt der behandelnde Arzt H. (vielleicht Adolf Haegler-Gutzwiller, 1830-1909) über die zum Tode führende Krankheit folgende vorsichtig formulierte Worte (S.687): «Der liebenswürdige Kranke, der dem Tode schon längst klar bewußt und standhaft ins Auge geblickt und die peinliche und für die Diagnose der Grundursache oft rätselhafte Krankheit mit charaktervoller Resignation und männlichem Muth ertragen hatte, blieb bis ans Ende freundlich und von rührender Liebenswürdigkeit für seine Umgebung und seine Freunde und dankbar für jede Liebeserweisung». Vorher ist von der Endocarditis, von den multiplen Embolien und von den Schädigungen der parenchymatösen Organe die Rede. Dem Wunsche Sondereggers, die Grabrede Willes nicht abzudrucken, war also Rechnung getragen worden. Dieser wichtige Brief Sondereggers zeigt seine Verehrung für Albert Burckhardt und seine human-verständnisvolle Einstellung aufs schönste. 
sehr beunruhigt. Ich bin Arzt, also gewiß nicht orthodox, aber dennoch frage ich: 1. wozu Dogmatik, positive und negative, von einem Arzte? 2. wozu negative Dogmatik bei einer Leichenfeier? 3. wozu Negation da, wo sie Niemandem nützt, Viele kränkt und einen sehr vernünftigen und taktvollen Freund noch post mortem auf die Bühne des müßigen Gezänkes zerrt! Ich bitte Sie um unsres lieben Burckhardt willen, ersparen Sie seinem Andenken diesen philosophischen Kuhreihen, denn mehr ist die Negation unter diesen Umständen nicht. Wie dem Andenken des Freundes, so schadet dieser dogmatische Cynismus auch dem Corr-Blatte und uns Ärzten. Wollen wir uns mit Tausenden ganz ohne Noth! verfeinden und in ihren Augen viel schlechter erscheinen als wir sind? Geburtshülfe in der Mädchenschule ist keine Wissenschaft, sondern eine Zote, die eine Maulschelle verdient und negative Dogmatik am Grabe, zumal am Grabe eines Freundes, ist auch eine solche Zote. Dem hochachtbaren Wille ist ein Lapsus passirt - nageln Sie ihn nicht fest! Scripta manent!

Schließlich lieber einen kurzen Zorn Willes als den Unwillen von $99 \%$ unserer Collegen. Ich gehöre auch zu diesen, nicht aus dogmatischen aber aus humanitären Gründen.

Auch scheint es mir, den stillen verläumderischen Gerüchten gegenüber, durchaus gut, ja nöthig, zu sagen, Burckhardt sei an Endocarditis Embolien und deren weiteren Folgen (ich würde sie kurz aufführen) gestorben. Das Schweigen über die Todesursache sieht ja ganz verdächtig aus, - in einem ärztlichen Journal!! Fragen wir nicht sogar bei Fernerstehenden: Was hat er gehabt? Sie schweigen? Ich frage als anständiger Mann nicht weiter, aber bin bitterlich überrascht und erstaunt. Armer Freund! Man darf deine Causa mortis gar nicht nennen! Hutten war ja ein großer Mann - aber sein "Leichenschein» ist uns doch ärgerlich. Wenn Burckhardt an Carcinom oder Tuberculose dachte, ist sein Wunsch begreiflich; so, wie die Section und wie die Fama steht, ist er unannehmbar, und um ihn auszuführen, muß man ihn umkehren. Er wollte sein Kind schonen und wir gehen mit boshaftem Gehorsam hin und - verläumden es. Bischoff ${ }^{30}$ ist krank, wenn er das nicht einsieht. Entschuldigen Sie mich. Der gute Zweck heiligt ja die Mittel, möge er auch diesen Brief heiligen.

$$
\text { Von Herzen Ihr }
$$

Sonderegger

\section{Lieber Freund!}

St. Gallen d 15 Dec 1886

Es ist eine schlimme Sache ums Corrigiren und Kritisieren; sehr oft verpfuscht man dabei viel. Ich habe mir einige kleine stylistische Änderungen erlaubt, - aber mit le i ch t a uswis chbarem Bleistift!

Man sieht der ganzen Rede den tiefgefühlten Schmerz und das Redliche Ehrliche des Lobes an, - auch den gemütlichen Druck unter welchem sie entstanden ist, und darum ist sie gut.

${ }^{30} \mathrm{Joh}$ ann Jakob Bischoff (1841-1892), Prof. der Geburtshilfe in Basel, trat 1887 wegen beginnender Geisteskrankheit zurück. 
Wenn Sie Willes Rede in Separatabzügen geben, so lassen Sie denno ch und e i n e we g den, unter gegebenen Umständen einfältigen Schluß weg! Unverschämtes Wort: «einfältig». Die Einen brauchen es nicht; die Andern werden tief, tief gekränkt und der Stand wird geschädigt. «Das sind sie, die Cyniker, die Nihilisten, die jedes Idealismus unfähigen Materialisten; das ist die gottlose Naturwissenschaft.» So wohlfeil lassen wir unsern Beruf nicht beschimpfen.

Wenn wir den Cultus der Materie lernen wollen, müssen wir ja zu denen gehen, welche sie am lautesten verläugnen - man kann das! Aber warum am Grabesrande, und am Grabe unseres tactvollen Freundes diese Taktlosigkeit!

Doch genug. Ich bin, wie ich hoffe, kein Heuchler, aber die Geschicht von der Geburtshilfe in der Mädchenschule kommt mir nicht aus dem Sinn.

In großer Eile, und mit herzlichen Grüßen

$\mathrm{Ihr}$

Sonderegger

Lieber Freund!

St. Gallen d 30 Decbr 86

Nehmen Sie beim Jahreswechsel meinen herzlichen Gruß, meinen warmen Dank und meine aufrichtigen Glückwünsche an! Wir sind über die Formalitäten hinweg, aber wir haben in diesem ablaufenden Jahre so Schweres gemeinsam gelitten, daß wir uns auch dadurch näher gekommen sind. Den Schmerz um Burckhardt werden wir ertragen, aber nicht verwinden und der edle liebe Mann wird mit uns leben, so lange wir leben und kämpfen. Sie haben bei diesem Andenken wenigstens ein gutes Gewissen, ich nicht; ich habe ihm zu wenig Liebe bewiesen, habe ihn sehr hoch geachtet, aber immer nicht hoch genug.

Schenken Sie mir ferner Ihre Freundschaft und Ihre Geduld und seien Sie vor Allem versichert, daß ich Sie nie mißverstehen werde, auch wenn Sie im Falle wären, mir energisch Opposition zu machen. Ich habe in Ihnen einen ehrlichen Freund gefunden und weiß ihn zu schätzen!

Nochmals herzlichen Dank für alle Ihre Liebe und Güte!

Ihr

Sonderegger

Lieber Freund!

St. Gallen d 6 Oct 87

Sie erhalten auch das Votum Schnyders. ${ }^{31}$ Wenn Sie damit einverstanden sind, lassen Sie es auch noch auf den 15. Nov. mitlaufen. In dieser Weise kann

${ }^{31}$ Ohne Zweifel denkt Sonderegger hier an den früheren Oberfeldarzt der Schweizerischen Armee K.Schnyder (1828-1900), der sich als Vorkämpfer für die Freizügigkeit der Ärzte und für die Zentralisation des Gesundheitswesens betätigt hat. Nach dem kurzen Nekrolog (Corr. Bl. 30, 1900, S. 454, f.) betrifft ein Antrag des Jahres 1887 (in einer Ärzteversammlung) diese letztgenannte Angelegenheit. Vgl. Sondereggers Brief vom 1. Nov. 1887. 
es angehen und es ist gut, wenn nicht nur wir beide "aufbegehren», sondern wenn auch andere die gleiche Melodie flöten.

Ihr

$$
\text { Dr. Sonderegger }
$$

NB. für die Correkturen erkläre ich mich persönlich verantwortlich.

DrS.

Lieber Freund!

St. Gallen d 26 Oct 87

Sie erhalten hier Ihre verschiedenen Belege. Gnehm ${ }^{32}$ perse ganz verschwiegen.

Über die Gesuche sprechen wir bei einer Ärzte Commission, die dieses Jahre noch sitzen muß. Ich wäre gegen Vorschüsse, Freiweg geben, oder nicht geben!

An Mercanton ${ }^{33}$ habe ich längst und ganz in Ihrem Sinne geschrieben und von der Versammlung der S. méd. romande «chaleureuses salutations»-Telegramm erhalten. Sie scheinen zufrieden gewesen zu sein. Die Schwierigkeit bleibt, unsere Leute über Bern hinaus zu bringen; sie gehen nicht und wir blamieren uns durch unsere schwache Betheiligung; hinc illae lacrymae m e a e ! Der Tagsatzungsabschied von 1829 enthält ein strenges Epidemienregulativ mit Cordons etc. Weitere $\mathrm{Org}$ a $\mathrm{n}$ is a t i onen kenne ich nicht. Die Abschiede sind in jeder Reg Räthl. Staatsbibliothek. Wenn ich gehe, muß Lotz ${ }^{34}$ oder Haffter ${ }^{35}$ dran. Wenn es keine Demüthigung ist, so sollten Sie Schriftführer des Centrl Ver. sein und bleiben. Wäre Ihr Ohrenleiden nicht, so müßten Sie der gegebene Praeses sein, das werden Sie bei aller Bescheidenheit nicht anzweifeln können.

Vergessen Sie den Becher ${ }^{36}$ nicht!

Auf glückliches Wiedersehen!

Ihr

Sonderegger

32 Ein Arzt namens $\mathrm{G} n$ e hm ist in den mir zugänglichen Nachschlagwerken nicht zu finden, auch im «Generalregister» des Correspondenzblattes fehlt eine entsprechende Angabe.

${ }_{33} \mathrm{Victor}$ Mercanton $(1850-1910)$, von Sonderegger auch in seinem «Lebensbild» (S.96) erwähnt, war Chirurg in Lausanne und sollte Sondereggers Nachfolge im Präsidium des «Ärztlichen Centralvereins» übernehmen, lehnte aber ab. ${ }^{34} \mathrm{Vgl}$. Anm. 11.

${ }_{35}$ Eli a s Ha f fter (1851-1909), nach Sonderegger die markanteste Gestalt im Standesleben der Schweizer Ärzte, auch Redaktor des «Correspondenzblattes» (1899-1909) und gewandter Schriftsteller, verdient auch durch die Herausgabe von Sondereggers Briefen.

36 Sonderegger spielt hier wohl auf den sogenannten «Vorpostenbecher»an, den er im Mai 1880 von der «Medizinischen Gesellschaft Basel» als Zeichen der Verehrung geschenkt erhielt. (Vgl. Sondereggers «Lebensbild» S. $66 \mathrm{f}$. und meine kurze Würdigung, Schweiz. med. Wschr. 27, 1143 XXXX [1946]). 


\section{Lieber Freund!}

In dem allgemeinen Trubel habe ich Sie beim Abschiednehmen verloren. Also hier noch nachträglich ein herzliches Adieu und «auf frohes Wiedersehen»! Nüt für ungut! mag auch berechtigt sein. Ich müßte demissionieren, weil ich mir selber vorwarf, bloß aus seniler Eitelkeit zu bleiben, und bloß zu schwatzen. Mit Zuwarten und langen Vorbereitungen wäre die an sich unbedeutende Sache schwieriger geworden. Kochers und Siggs ${ }^{37}$ demonstrative Abreisen sind charakteristisch. Nach meiner Meinung ist die Wahl Haffters sehr gut; er ist «Arzt» und «hats an den Leuten.» Die Cassierfragen regeln wir bei Anlaß der Arzte Commission. Der große und wichtige Cassier sind Sie, und Ihre Stelle ist formell richtig besetzt worden. Die andern Kleinigkeiten kann Jeder verwalten.

Ebenso sind Sie Actuar des Centralvereins, d. h. Staatskanzler, der den neuen Praeses ganz sachte und ganz collegial einführt.

Mit Schnyder ${ }^{38}$ stehe ich in Unterhandlung - er möge seinen Antrag zurückziehen, beharrt er auf der Mittheilung, so werde ich ihn sofort bekämpfen und bitte ich um ein paar höfliche Zeilen in derselben Numero des Corr. Blattes. Diesen doctrinären Unsinn sollten wir gar nicht öffentlich besprechen. Bundesverfassungsrevision? bloß wegen der Döcter? meinetwegen «bloß wegen des Sanitätswesens?» Da muß man doch, so wie die Sachen jetzt stehen, mit Glanz abfahren, und dann kann man ein paar Jahre gar nicht mehr damit kommen.

Den Toast habe ich an Munzinger ${ }^{39}$ junior, auf dessen Verlangen geschickt. Sie werden ihm einigen Ernst ansehen. Ich hatte ihn zu Olten gar nicht bei mir.

Puncto Wien ${ }^{40}$ will ich Ihnen später Nro II liefern, ein Résumé aller 25 Thema-Resolutionen; aber erst wenn der officielle Text herausgekommen ist.

Mit herzlichen Grüßen und mit der Bitte um alte Geduld und Freundschaft

Ihr

$$
\text { Dr. Sonderegger }
$$

37 Vgl. dazu Anm. 12 und 15.

38 Vgl. dazu Anm. 31.

39 Wahrscheinlich meint Sonderegger den Solothurner Arzt Eugen Mun . zinger (1830-1907), Chefarzt am Spital Olten, der sich auch als Dichter einen Namen machte.

40 Als Delegierter des Bundesrates besuchte Sonderegger (mit Fridolin Schuler) zusammen den VI. internationalen Hygiene-Kongreß in Wien im Sommer 1887. Er berichtet in eindrucksvoller Weise über den dabei entfalteten Prunk («Lebensbild», S. $81 \mathrm{ff}$.). 


\section{Lieber Freund!}

Schnyder ${ }^{41}$ hat etwas Wasser in seinen Wein gegossen, so können wir ihn schon präsentiren. Eine Revision der B. Verf. Art 33 und 69 «nur für die Döcter» zu verlangen, wäre Unsinn; wenn wir bei Anlaß sonstiger Revision damit kommen wollen, vergeben wir uns nichts; wir müssen nur fürsorgen, daß unsere Begehren niemals einem Schutzzoll für die Ärzte gleichsehen. Wir haben ein gutes Gewissen und dürfen den bösen Schein wohl meiden.

Finden Sie Schnyders Vorschlag auch in dieser Form noch zu irritirend, so sagen Sie es mir, ich habe von Schnyder blanco Credit zu ändern wie ich will.

Mit herzlichen Grüßen Ihr

$$
\text { Dr. Sonderegger }
$$

\section{Lieber Freund!}

St Gallen d 21 Dec 87

Gestatten Sie mir, Ihnen einen kleinen symbolischen Gruß unter Ihren Weihnachtsbaum zu legen. Möge die Reise durch das neue Jahr für Sie eine glückliche und möge Ihr Gepäk niemals ein sorgenschweres sein! Einsam stehend, sind Sie ein lieber Familienvater für Ihre Neffen ${ }^{42}$ und für viele, die Hülfe und Trost suchen. Möge auch dieses herzerquickende Wirken Ihnen wieder wohl gelingen!

Wenn ich Sie je gekränkt habe, verzeihen Sie mir, meine Absichten waren immer redliche und meine freundschaftliche Hochachtung ist eine alte, unwandelbare!

Ihr Dr. Sonderegger

\section{Lieber Freund!}

St Gallen d 2 Jan 1888

Meine herzlichen Glückwünsche zum neuen Jahr! Daß Sie am Grabe Burckhardts meiner gedacht, thut mir wohl, und ich danke es Ihnen herzlich. Sie glauben an meine treue Liebe und Hochachtung für den Entschlafenen.

Und wie "wir werden uns weniger oft sehen als bisher»? ${ }^{43}$ Das ist mir un-

$41 \mathrm{Im}$ Register des Correspondenzblattes ist kein ähnlich lautendes «Votum» Schnyders verzeichnet. Vgl. jedoch Anm. 31 .

${ }^{42}$ Mit Bleistift ist offenbar von einer späteren Besitzerin der Briefe zum Worte «Neffen» hinzugesetzt: «soll heißen: Nichten!»

43 Offenbar spielte Baader in dem von Sonderegger zitierten Passus auf seine Krankheit an. Er starb am 25. Februar 1888 an einer kruppösen Pneumonie. In seinem Gedenkwort an Baader (Corr. Bl. 18, 1888, S. 130) schwebt Sonderegger wohl die zitierte Stelle vor, wenn er sagt, Baader sei schon seit einigen Wochen von einer tiefen Resignation erfüllt gewesen. Baaders Gesundheit war durch seine aufreibende Tätigkeit schon früher ins Wanken gebracht worden. Im Jahr 1877 hatte er eine Hämoptoë erlitten (vgl. Sondereggers Brief vom 29. März 1877, s. oben S. 199) ; darauf hatte er die Land- mit der Stadtpraxis in Basel vertauscht. 
verständlich, die Ärzte Commission liquidiert ihr Geschäft nicht und wir werden genau so oft und in jeder Beziehung so intensiv miteinander verkehren wie bisher.

Garrès ${ }^{44}$ Wegzug thut mir sehr leid für Sie und ich wünsche Ihnen Glück beim Aufsuchen seines Nachfolgers. Allein werden Sie auch diese Last, zu allem Andern, nicht auch tragen wollen.

Seien Sie mir herzlich und in alter Freundschaft gegrüßt!

Ihr

Dr. Sonderegger

Für Ihre schönen Briefpapiere herzlichen Dank von der fleißigen Nièce. ${ }^{45}$

Lieber Freund!

St Gallen d 9 Jan 88

Ich bitte Sie, mir das Manuscript wegen des Concordates mit England, ${ }^{46}$ innert 4 Tagen zukommen zu lassen; wäre es schon vernichtet, dann $1-2 \mathrm{Ab}$ züge auf gutem Schreibpapier. Ich schicke das Ding nach Bern, wo große Gefahr ist, vom drängenden England über den Löffel balbiert zu werden.

Nun noch Eines:

Welches Arbeitsprogramm würden S i e entwerfen für einen Eidg. Medicinal Referenten oder «Gesundheitssekretär»? Ich will nicht meine Privatmeinungen verzapfen, sondern mit Ihnen und einigen andern erfahrenen Collegen Fühlung behalten. ${ }^{47}$

In herzlicher Hochachtung

Ihr alter Sonderegger

Lieber Freund!

St Gallen d 12 Januar 1888

Ich habe noch eine Menge Data nachgeholt, doch fehlen noch 25! Der Setzer

" C a r l G a r r è (1857-1928), von 1886-1888 Dozent für Chirurgie und Bakteriologie in Basel, kurze Zeit auch Redaktor des Correspondenzblattes», ging als Oberarzt zu P. v. Bruns in Tübingen. Letzteres Fach, das Garrè als erster in Basel lehrte, lag Sonderegger besonders am Herzen. Von 1890 an lehrte Albert Dubler (1857-1903) pathologische Anatomie und Bakteriologie.

4. Mit der «N i è c e » ist nach den freundlichen Angaben von Herrn Dr. P. Jung wahrscheinlich Frau Knöpfel in Heerbrugg, vielleicht aber auch eine Tochter aus der näheren Bekanntschaft gemeint.

46 «Concordat mit England», worum es sich hier handelt, ist mir nicht bekannt. Auch Herr Dir. P. Vollenweider, Vorsteher des eidgenössischen Gesundheitsamtes, konnte mir darüber nichts Näheres mitteilen. Für seine freundlichen Auskünfte sei ihm hier bestens gedankt.

${ }_{47}$ Im April 1889 faßte der Bundesrat, wie Sonderegger in seiner Autobiographie (S. 88 f.) schreibt, den Beschluß, einen Sanitätsreferenten zu ernennen, 1893 (28. Juni) «wurde das eidgenössische Gesundheitsamt geschaffen» (ebda. S.95). 
kann das mit Muße nachtragen. Fort mit der Fußnote am Anfang! Der werthe Dr. Sonderegger soll hinter den Coulissen bleiben!

Ich hoffe schließlich doch noch Alles zu füllen. Nur Rob Koch. 11 Dec 1843 habe ich direkt geschrieben.

\section{Herzliche Grüße!}

$$
\text { Dr. Sonderegger }
$$

Herrn W. Baader Basel ${ }^{48}$

Verehrter Herr!

St. Gallen d. 1. März 1888

Empfangen Sie nochmals meine warme Condolenz. Wir sind in aller Form verwaist, denn der liebe Doktor war ein Vater für Viele und für Vieles "was Menschenherz erhebt». Noch unmittelbar ehe er erkrankte, schrieb er mir nebenbei «der olle Knabe wird nicht mehr lange Berichte lesen.» Er hat sein Schicksal kommen gesehen und es mit der Ruhe des Weisen erwartet. Es ist sehr dafür gesorgt, daß wir den lieben Freund nicht vergessen, so lange wir leben.

Nehmen Sie es mir nicht als Rohheit, daß ich die Beerdigungsfeierlichkeit nicht mitgemacht. Ich fehlte auch bei Burckhardt Merian, bei Horner und bei Vielen, die mir nahe standen. Meine Grabrede hielt ich im Corr Blatt, zu Handen der Berufsgenossen; vor einem mir unbekannten Publikum kann und will ich nicht reden über einen Todten, dessen Name und Andenken mir heilig ist.

Sie haben sehr viel verloren, verehrter Herr! und haben in diesen Tagen den Schmerz um Ihre liebe Mutter und um Ihren trefflichen Vater aufs neue durchgearbeitet. Möge Ihnen Trost und Resignation beschieden sein!

In Hochachtung grüßend

Ihre ergebener

Dr. Sonderegger

Herrn W. Baader,

hiemit die ergebene Mittheilung, daß ich Herrn Physicus Dr. Lotz gebeten habe, die Hülfskasse zu übernehmen und meinen warmen Dank für alle wohlwollenden Bemühungen in dieser Sache!

In Hochachtung ergeben:

St. Gallen d. 1. März 1888

$$
\begin{aligned}
& \text { D r. Sond e r e g g e r } \\
& \text { Pt. d. Schwz. } \\
& \text { Ärzte Comm. }
\end{aligned}
$$

48 Als Abschluß der vorliegenden Reihe von Briefen Sondereggers sei sein Kondolenzschreiben an den Bruder Arnold Baaders, den Kaufmann Walter Baader (1844-1910), beigefügt, da in diesem die Freundschaft des St. Galler Arztes mit dem Basler Kollegen noch einmal sehr schön zum Ausdruck kommt. 